\title{
RIO-DC Buffer Design for Core Routers in DiffServ Assured Services
}

\author{
Kyeong Hur, Member, KIMICS
}

\begin{abstract}
In this paper, a parameter optimization method of RIO-DC (RED (Random Early Detection) with In and Out-De-Coupled Queues) scheme for Assured Service (AS) in Differentiated Services (DiffServ) is proposed. In order to optimize QoS (Quality of Service) performance of the RIODC policy for AS in terms of maximum tolerable latency, link utilization, fairness, etc., we should design router nodes with proper RIO-DC operating parameter values. Therefore, we propose a RIO-DC configuration method and the admission control criterion, considering the allocated bandwidth to each subclass and the corresponding buffer size, to increase throughput for In-profile traffic and link utilization. Simulation results show that RIO-DC with the proposed parameter values guarantees QoS performance comparable with the RIO scheme and it improves fairness between AS flows remarkably.
\end{abstract}

Index Terms- RIO-DC, Assured Service, Differentiated Services, Fairness, and QoS.

\section{INTRODUCTION}

WITH the proliferation of multimedia and real time applications, it is increasingly becoming more important to guarantee certain QoS for Internet applications. The DiffServ model has been proposed as a scalable way of providing QoS in the Internet [1], [2]. Scalability is achieved by moving complicated functionalities such as per-flow or flow aggregate marking, shaping, and policing to the edge routers and by leaving the core routers with very simple functionality. With DiffServ, the core router treats packet forwarding according to a DiffServ codepoint (DSCP) marked at the edge router, where a DSCP corresponds to a Per-Hop Behavior (PHB). DiffServ provides packet level service differentiation through the simple and predefined PHBs.

The IETF has defined Expedited Forwarding (EF) PHB for Premium Service (PS) and Assured Forwarding (AF) PHB for Assured Service (AS) respectively [3], [4]. Since the EF PHB was introduced to provide low loss, low latency, and assured bandwidth for supporting real time

Manuscript received July 11, 2011; revised August 10, 2011; accepted August 18, 2011.

Kyeong Hur is the corresponding author with the Department of Computer Education. Gyeongin National University of Education, Incheon, Korea(e-mail: khur@ginue.ac.kr). services such as IP telephony, video conferences and the like, it is expected that the PS would be assigned to a high-priority queue in the routers. Since the AF PHB was defined to provide different levels of forwarding assurances according to the customer's profile [4], it was proposed to use the RIO approach to ensure the "expected capacity" specified by the service profile for AS. Upon each packet arrival, if the traffic rate is within the subscribed traffic profile, the packet is marked as "Inprofile". Otherwise, it is marked as "Out-of-profile". In a DiffServ aware router, all the incoming packets are queued in the original reception order. However, during network congestion, the router preferentially drops the packets that are marked as "Out-of-profile". By appropriate provisioning, if we could make sure that the aggregate "In-profile" packets would not exceed the capacity of the link, the throughput of each flow or flow aggregate could be assured to be at least the rate defined in the contracted traffic profile.

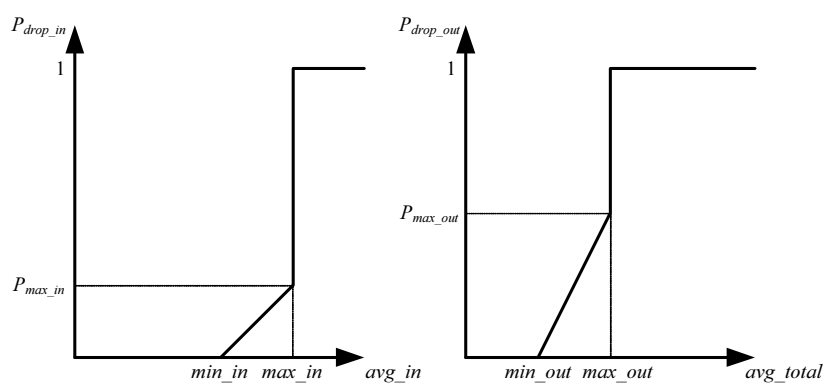

(a)

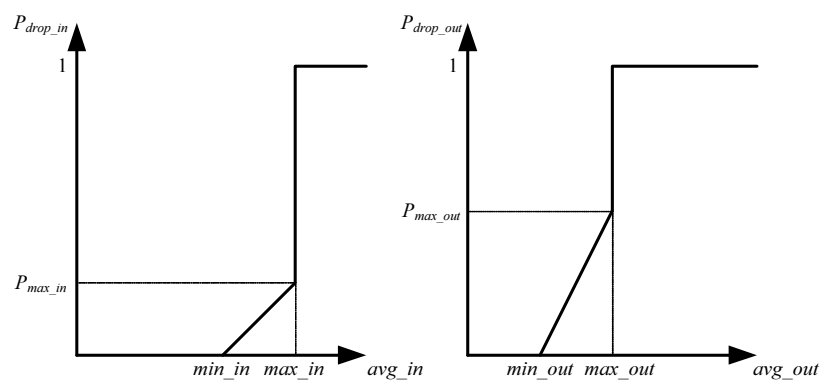

(b)

Fig. 1. Buffer management policy of RIO (a) / RIO-DC (b). 
As shown in Fig. 1(a), the RIO scheme drops incoming In-profile packets according to the average number of Inprofile packets (avg_in) and it drops incoming Out-ofprofile packets according to the average number of total packets in the queue (avg_total).

Fig. 1(b) shows the RIO-DC scheme that drops incoming In-profile packets according to the average number of In-profile packets ( $a v g$ in) and it drops incoming Out-of-profile packets according to the average number of Out-of-profile packets in the queue (avg_out).

Since the RIO-DC scheme calculates the average number of packets per each virtually de-coupled queue and drops the Out-of-profile packets according to the decoupled avg_out value, In-profile packets can be more prioritized when we apply the RIO-DC scheme instead of RIO into a queue. In Fig. 1, it is not difficult to anticipate that performance of the RIO/RIO-DC operation depends on the configuration parameters such as max_in, max_out, min_in, and min_out. In order to guarantee the expected capacity for the In-profile packets and maximize overall link utilization in the applied buffer management policy, the configuration parameters should be optimized to effectively drop the packets beyond the service profile in case of network congestion. In [5], we've optimized the RIO operating parameters and demonstrated its outstanding performance. Therefore, in this paper, we propose an optimization scheme for the RIO-DC parameters which differentiates allocated resource per each AS subclass and guarantees fairness in throughput performances of In-profile packets among AS flows.

This paper is organized as follows: In Section II, we describe the proposed RIO-DC configuration optimization scheme. In Section III, simulations are executed and their results are discussed. Finally, in Section IV, concluding remarks are presented.

\section{PARAMETER OPTIMIZATION OF RIO-DC FOR ASSURED SERVICE}

The characteristic of the AS In-profile traffic can be defined with average/maximum subscribed data rate and burst length at a leaf router in a DiffServ domain [6]. When a user subscribes average data rate $r$ and maximum peak data rate $p$ for a network service, the maximum delay time for $j$ th subclass with over-provisioning factor $\mu_{j}$ in AS can be guaranteed as follows:

$$
d_{\text {max }, j}=\frac{\left(b-\mu_{j}\right) \cdot l}{\mu_{j}}
$$

where $b=p / r, l$ is a burst length, and the range of $\mu_{j}$ is from 1 to $b$ (if $\mu_{j}=b$, then PS). In this paper, since we assume that $\mu_{j}=1$ where only one subclass is defined for
AS, the maximum delay of $(b-1) \cdot l$ can be guaranteed for In-profile packets.

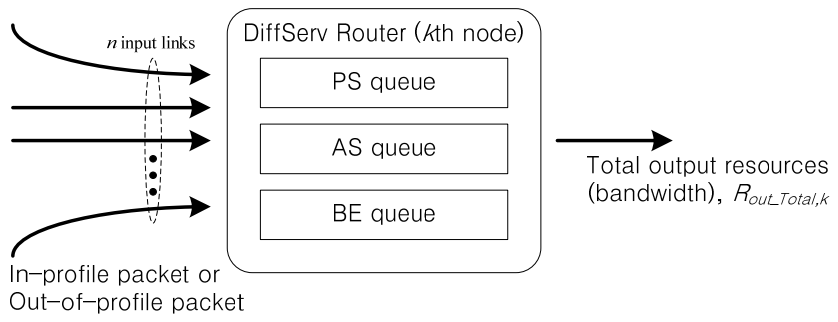

Fig. 2. DiffServ Router .

At the $k$ th node, the AS traffic resource can be defined as in (2) considering the reserved resource for the PS traffic, $R_{\text {out_PS, } k}$.

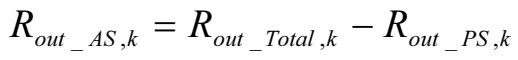

Assuming $n$ input links to $k$ th router node, the ratio between the input and output link bandwidth $\lambda_{\text {in/out }}$ can be described as in (3).

$$
\begin{aligned}
\lambda_{\text {in/out }}= & \frac{R_{\text {in_AS }, k}}{R_{\text {out_AS, } k}}=\frac{\sum_{i=1}^{n} R_{\text {out_Total }, i}-R_{\text {out_PS }, k}}{R_{\text {out_Total }, k}-R_{\text {out }_{-} P S, k}}, \\
& \text { where } R_{\text {out_ } P S, k}=\sum_{i=1}^{n} R_{\text {out }_{-} P S, i}
\end{aligned}
$$

If we determine the max_in and max_out of the RIODC keeping the ratio between the amount of the In-profile packets and that of the Out-of-profile packets to $1: \beta(\beta<<$ 1 ) in order to make the In-profile packets use most of resources of an output link, the resource utilization ratio between them can be guaranteed as $1: \beta$. Therefore, the reserved output link resources for the In-profile packets and those for the Out-of-profile packets are equal to $R_{\text {out_AS, }, k} /(1+\beta)$ and $R_{\text {out_AS, }, k} \beta /(1+\beta)$, respectively.

In addition, we should keep the summation of the average transmission rates of $n$ In-profile packet flows below the reserved resources for them as in (4) to maintain the resource utilization ratio.

$$
\mu_{j} \sum_{i=1}^{n} r_{i} \leq \frac{R_{\text {out_AS, }, k}}{1+\beta}
$$

Assuming $\mu_{j}=1$, the maximum number of the Inprofile packets and that of the Out-of-profile packets arrived from the previous router node are $R_{\text {out } \_A S, k} \cdot b_{\text {max }} \cdot l_{\text {max }} /\{(1+\beta) \cdot$ PacketSize $\} \quad$ and $\beta$. $R_{\text {out } A S, k} \cdot b_{\max } \cdot l_{\max } /\{(1+\beta) \cdot$ PacketSize $\}$, respectively, where $b_{\max }$ and $l_{\max }$ are the maximum ones among the subscribed 
$b_{i} \mathrm{~s}$ and $l_{i} \mathrm{~s}$ during $\tau$ period. Since $\lambda_{\text {in/out }}<b_{\max }$ in normal cases, those can be modified as follows: $R_{\text {out_AS, } k} \cdot \lambda_{\text {in/out }} l_{\max } /\{(1+\beta) \cdot$ PacketSize $\} \quad$ and $\beta \cdot R_{\text {out_AS, } k} \cdot \lambda_{\text {in/out }} l_{\text {max }} /\{(1+\beta) \cdot$ PacketSize $\}$. Accordingly, the maximum number of the remained In-profile packets and Out-of-profile packets in the buffer are equal to $R_{\text {out_AS, }, k} \cdot\left(\lambda_{\text {in/out }}-1\right) \cdot l_{\text {max }} /\{(1+\beta) \cdot$ PacketSize $\} \quad$ and $\beta \cdot R_{\text {out } A S, k} \cdot\left(\lambda_{\text {in/out }}-1\right) \cdot l_{\max } /\{(1+\beta) \cdot$ PacketSize $\}$.

In [5] and [7], a criterion for the RIO parameters has been defined as follows:

Criterion I-1: The max_in and max_out of the RIO scheme are determined as the maximum available buffer size for the In-profile packets and total packets, respectively.

In case of the RIO-DC policy, the operating parameters should be determined based on the criterion described below according to the definition of RIO-DC scheme:

Criterion I-2: The max in and max_out of the RIO-DC scheme are determined as the maximum available buffer size for the In-profile packets and Out-of-profile packets, respectively.

Therefore, we propose the optimized values of the max_in and max_out in Table 1 where taking $\mu_{j}$ into account.

TABLE I.

PROPOSED VALUES FOR THE RIO/RIO-DC PARAMETERS

\begin{tabular}{|l|c|}
\hline \multicolumn{1}{|c|}{ Parameter } & \multicolumn{1}{|c|}{ Value } \\
\hline $\begin{array}{l}\text { max_in of } \\
\text { RIO } / \text { RIO- } \\
\text { DC }\end{array}$ & $\frac{R_{\text {out }_{-} A S, k} \cdot\left\{\min \left(\lambda_{\text {in } / \text { out }}, b_{\max }\right)-\mu_{j}\right\} \cdot l_{\max }}{\mu_{j} \cdot(1+\beta) \cdot \text { PacketSize }}$ \\
\hline $\begin{array}{l}\text { max_out of } \\
\text { RIO }\end{array}$ & $\frac{R_{\text {out }_{-} A S, k} \cdot\left\{\min \left(\lambda_{\text {in } / \text { out }}, b_{\max }\right)-\mu_{j}\right\} \cdot l_{\max }}{\mu_{j} \cdot \text { PacketSize }}$ \\
\hline $\begin{array}{l}\text { max_out of } \\
\text { RIO-DC }\end{array}$ & $\frac{\beta \cdot R_{\text {out }_{-} A S, k} \cdot\left\{\min \left(\lambda_{\text {in } / \text { out }}, b_{\max }\right)-\mu_{j}\right\} \cdot l_{\max }}{\mu_{j}(1+\beta) \cdot \text { PacketSize }}$ \\
\hline
\end{tabular}

In addition, in order to determine the value of $\beta$ with improving link utilization, we introduce another criterion for the RIO-DC parameter optimization as in [5].

Criterion II: When the In-profile packets do not exist, the output link resources should be used fully only with the Out-of-profile packets.

Thus, we propose to set $\beta$ according to the Criterion II as shown in (5).

$$
\beta=\frac{\mu_{j}}{\min \left(\lambda_{\text {in } / \text { out }}, b_{\text {max }}\right)-\mu_{j}}
$$

In general, the min in and min_out can be determined as the half values of the max_in and max_out, respectively [7].

Finally, we can introduce an efficient configuration framework for the RIO-DC scheme in Fig. 3. Fig. 3 shows the operational flow of the RIO-DC engine for $k$ th router. Therefore, the operational parameters such as max_in, max_out, min_in, min_out and $\beta$ are optimized using the operation described in Fig. 3.

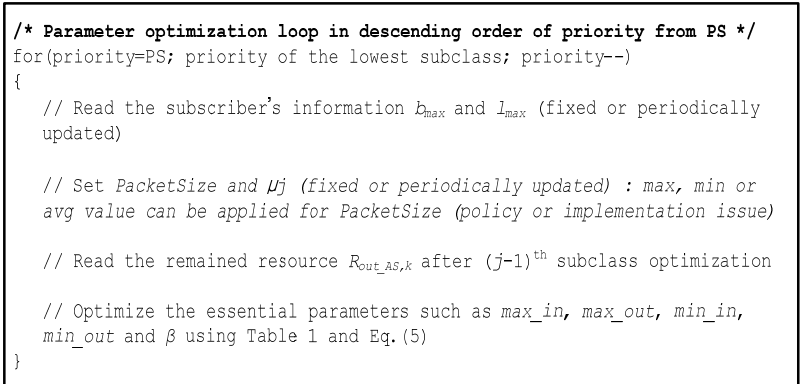

Fig. 3. The operational flow of the RIO-DC engine.

\section{SIMULATION RESULTS}

Performance of the proposed algorithm is evaluated via ns2 simulations. In the simulation, we assume that the over-provisioning factor $\mu_{j}=1$ and each host has the same traffic profile (i.e., average and maximum peak data rate). Also, the same RTT (Round Trip Time) and the same number of hops are assumed to minimize the effect of Token loss at a router caused by missed ACKnowledgements which might be dropped due to the difference in RTTs or the number of hops.

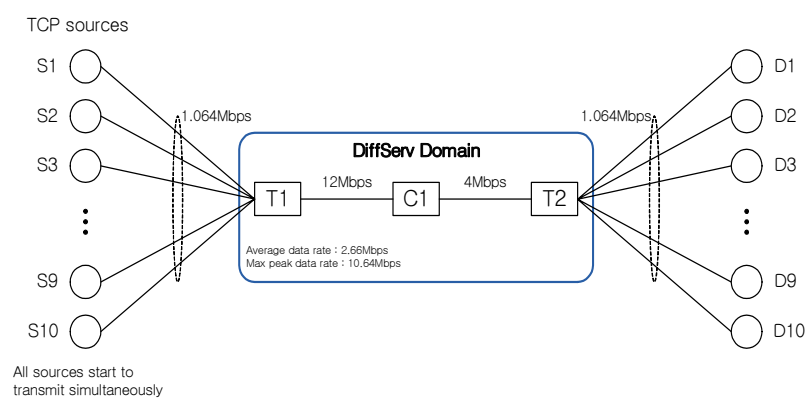

Fig. 4. The simulation model $\left(b_{\max }>\lambda_{\text {in/out }}\right)$.

Fig. 4 shows the network topology used in the simulation. Each host $S n$ sends the corresponding destination node $D n$ data with the average rate of $0.266 \mathrm{Mbps}$ and the maximum peak rate of $1.064 \mathrm{Mbps}$. In the DiffServ domain, the $T 1$ and $T 2$ are terminal DiffServ routers and the $C l$ is a core DiffServ router. Thus, average $2.66 \mathrm{Mbps}$ and maximum $10.64 \mathrm{Mbps}$ traffic packets are arrived from 10 hosts and the packets pass the 
C1 router through the $12 \mathrm{Mbps}$ input link and the $4 \mathrm{Mbps}$ output link. Table 2 summarizes other simulation parameters on the simulation model in Fig. 4.

Accordingly, the required buffer size for max_out can be determined using Table 1 as 264 packets * PacketSize for RIO and 88 packets * PacketSize for RIO-DC, respectively. That is, the max_in and max_out are set to 176 and 264 for RIO and 176 and 88 for RIO-DC.

TABLE II.

THE SIMULATION PARAMETER CONFIGURATION IN FIG. 4.

\begin{tabular}{|c|c|c|c|c|c|c|}
\hline RTT & $L_{\text {MAX }}$ & $\begin{array}{c}\text { PACKET } \\
\text { SIZE }\end{array}$ & $P_{\text {MAX_IN }}$ & $P_{\text {max_out }}$ & $W_{Q}$ & $A_{\text {IN OUT }}$ \\
\hline $16 \mathrm{~ms}$ & $\begin{array}{c}33 \mathrm{~m} \\
\mathrm{~S}\end{array}$ & $125 \mathrm{bytes}$ & 0.02 & 0.05 & 0.002 & 3 \\
\hline
\end{tabular}

Fig. 5 shows the variation of the number of the arrived packets and the departed ones within 100 seconds at the C1 router according to each max in and max out value. As shown in Fig. 5, the optimized values of the max in and max out of RIO, i.e., $\max$ in $=176$ and max out $=264$ make the almost best performance and those of the max_in and max_out of RIO-DC, i.e., $\max i n=176$ and max out $=88$ show the similar performance. Since the RIO-DC engine manages its buffer according to the number of Out-of-profile packets in the corresponding virtually de-coupled queue, we can see that the throughput of the In-profile packets highly depends on its max_out value. The number of passed packets at the $\mathrm{C} 1$ router at each RIO/RIO-DC parameter value is shown in Fig. 6. With the proposed values, we can get $99.875 \%$ and $99.2 \%$ link utilization rates for the RIO and the RIO-DC respectively, and these results are approximately the best performance. Therefore, the proposed parameter optimization scheme can optimize the throughput performance of the In-profile packets and the link utilization.

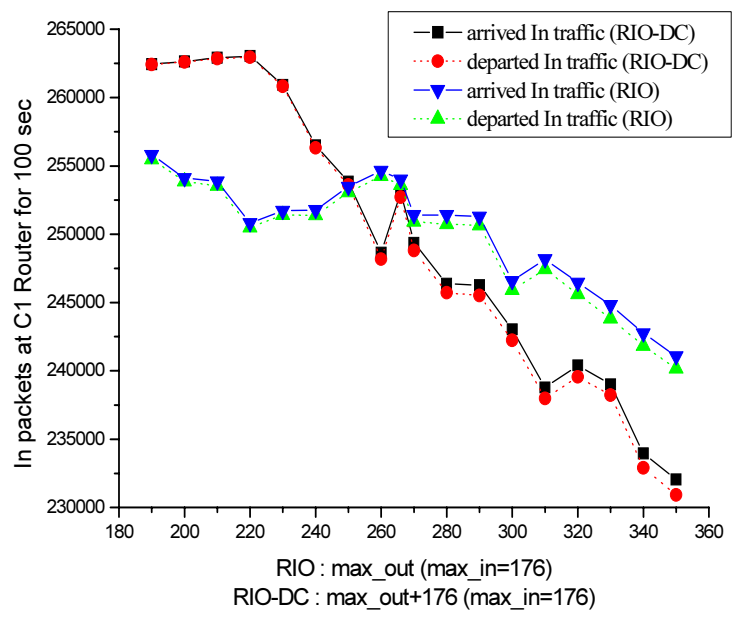

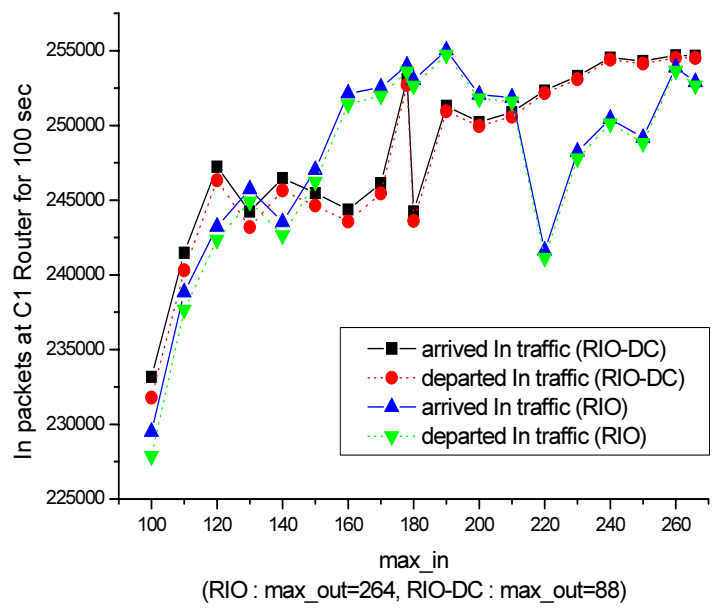

Fig. 5. The number of the arrived packets and the departed ones at the $C 1$ router.
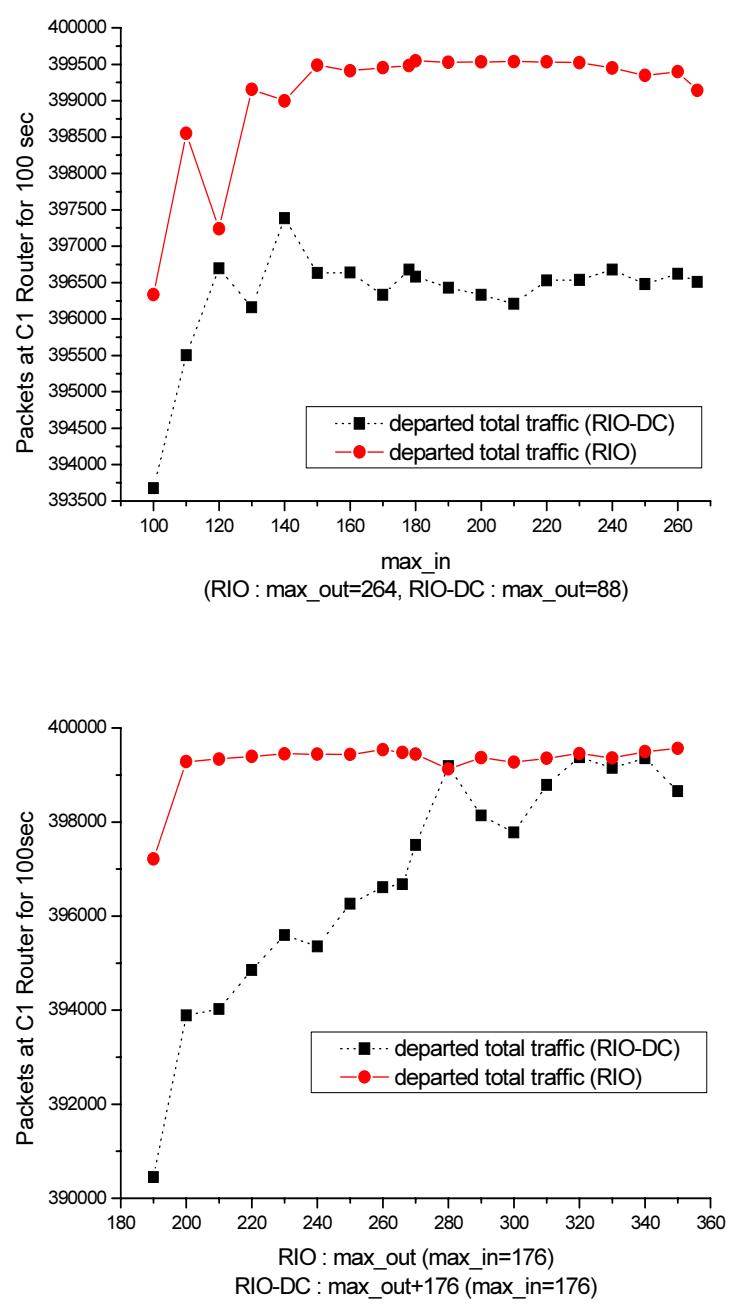

Fig. 6. The number of the passed packets at the $C 1$ router. 
The average queue length variation at the $\mathrm{C} 1$ router is shown in Fig. 7. Since the traffic characteristics are same each other, the average queue length variation is very small. Both the avg_in and avg_total values for RIO and those values for RIO-DC are near around the min in and min_out respectively. Thus, the simulation results show the same performance of the In-profile traffic throughput and the total link utilization.
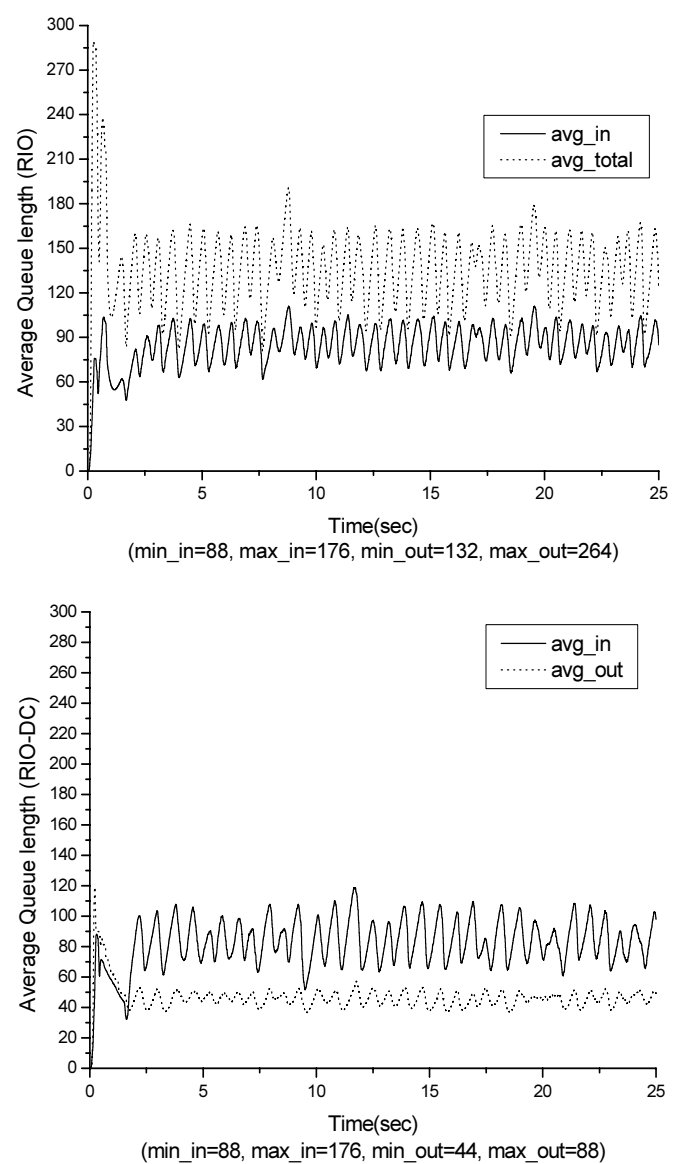

Fig. 7. Average queue length variation at the $C 1$ router.

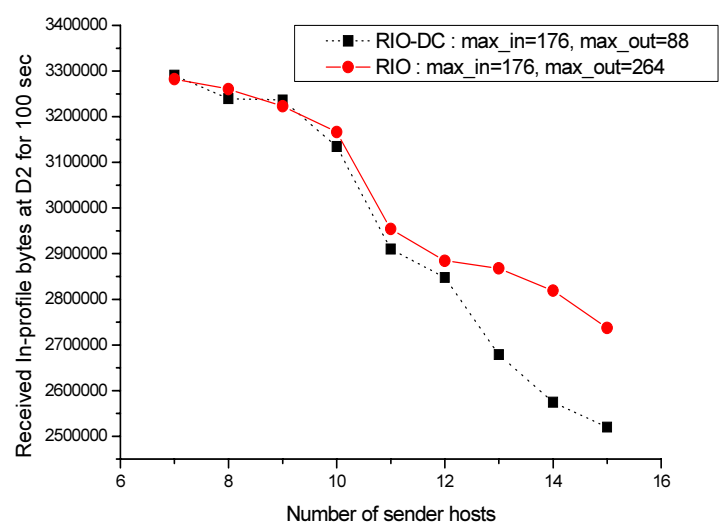

Fig. 8. The received bytes of the In-profile packets at the D2 according to the number of the sender hosts.
Fig. 8 shows the received bytes of the In-profile packets per 100 seconds at the D2 node in Fig. 4 according to the number of the sender hosts. For this simulation, we assume 15 hosts with the average data rate of $0.266 \mathrm{Mbps}$ to make the In-profile packets fully utilize 4Mbps output link of the $\mathrm{C} 1$ router. As shown in Fig. 8, the received bytes cannot be reached to the maximum value of 3325000 bytes $(0.266 \mathrm{Mbps} * 100 \mathrm{sec})$ even when the number of the sender hosts is very small. It might be occurred by the Token loss caused by the Early Random Drop. With the proposed optimum values for both schemes, the contracted throughput for the In-profile packets can be guaranteed up to 10 sender hosts which is the assumed condition for the proposed values. Therefore, the admission control based on the proposed parameter configuration scheme gratifies the QoS requirement and gets the maximum capacity.

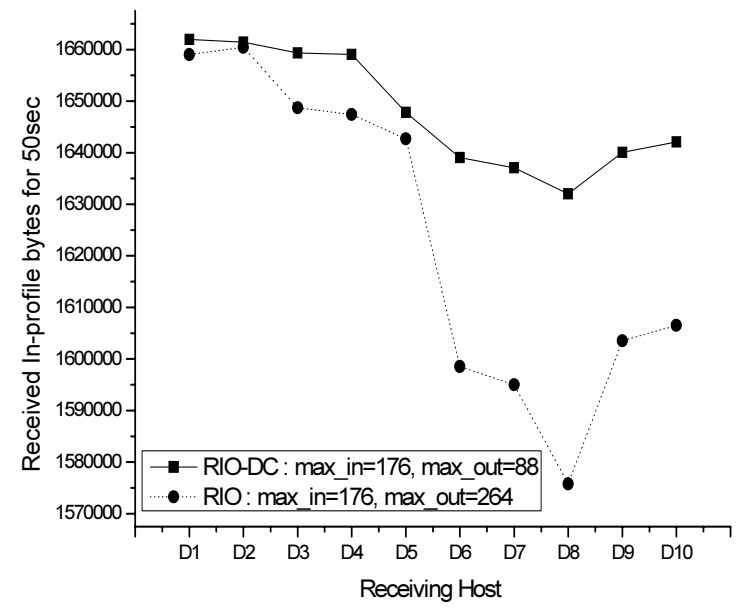

Fig. 9. The received bytes of the In-profile packets at each Dn

In order to compare fairness performance, we let each sending host starts to transmit data one after another at every 10 seconds, e.g., $S 1$ sends its data at 0 second, $S 2$ starts to send after 10 seconds, etc. Since RIO policy drops Out-of-profile packets according to the value of avg_total, In-profile packets which are sent later might be delayed or dropped if the Out-ofprofile packets which have already arrived at the $C 1$ router occupy the buffer of $C 1$ router more than $\beta$ ratio. However, the virtually de-coupled queue of RIO-DC scheme prevents that problem by managing the Out-ofprofile packets directly based on the value of independent avg_out. Accordingly, the simulation results in Fig. 9 show the fairness guaranteed performance of RIO-DC algorithm. 


\section{ACKNOWLEDGMENT}

This work was supported in part by Basic Science Research Program through the National Research Foundation of Korea (NRF) funded by the Ministry of Education, Science and Technology (MEST) (20100002366) and in part by Mid-career Researcher Program through NRF grant funded by the MEST (2011-0016145).

\section{CONCLUSIONS}

In this paper, a novel configuration optimization process of the RIO-DC operating parameters has been proposed for efficient DiffServ router operations. The proposed scheme can differentiate the allocated resource per each AS subclass and can improve output link utilization. Furthermore, it can guarantee certain level of fairness. Such a configuration optimization mechanism will play a key role in a DiffServ network implementation which has QoS requirements such as throughput performance, fairness, etc. Since the additional overhead for the computation is very small, the proposed method can easily be applied to the DiffServ network design.

\section{REFERENCES}

[1] M.Carlson, et. al., "An Architecture for Differentiated Services," RFC 2475, December 1998.

[2] Xipeng Xiao and Lionel M. Ni, "Internet QoS: A Big Picture," IEEE Network, pp. 1234-1250, March/April 1999.

[3] V. Jacobson, K. Nichols, and K. Poduri, "An Expedited Forwarding PHB," RFC 2598, June, 1999.

[4] J. Heinanen, F. Baker, and et. al., "Assured Forwarding PHB Group," RFC 2597, June, 1999.

[5] Y. -I. Joo, K. Hur, J. -K. Kim, D. -S. Eom and Y. Lee, "RIO Configuration Optimization for Assured Service in DiffServ Networks," IEEE Transactions on Consumer Electronics, Vol. 55, No. 4, pp. 1968-1972, Nov. 2009.

[6] Ilias Andrikopoulos and et. al., "A Fair Traffic Conditioner for the Assured Service in a Differentiated Service Internet," Proceedings of ICC 2000, pp. 806-810, June 2000.

[7] S. Floyd, V. Jacobson, "Random Early Detection gateways for Congestion Avoidance," IEEE/ACM Transactions on Networking, vol. 1, no.4, pp. 397-413, August 1993.

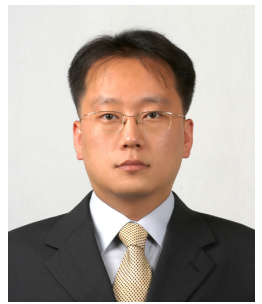

Kyeong Hur (corresponding author) is currently an Assistant Professor in the Department of Computer Education at Gyeongin National University of Education, Republic of Korea. He was senior researcher with Samsung Advanced Institute of Technology (SAIT), Korea from September 2004 to August 2005. He received a M.S. and Ph.D. in Department of Electronics and Computer Engineering from Korea University, Seoul, Korea, in 2000 and 2004, respectively. His research interests include; computer network designs, next generation Internet, Internet QoS, and future All-IP networks. 